\title{
openheart Exercise prescription improves exercise tolerance in young children with CHD: a randomised clinical trial
}

\author{
Sinead Callaghan (10 ,1,2 Margaret Louise Morrison, ${ }^{1}$ Pascal P McKeown, ${ }^{1,2}$ \\ Christopher Tennyson, ${ }^{1}$ Andrew J Sands, ${ }^{1}$ Brian McCrossan, ${ }^{1}$ Brian Grant, ${ }^{1}$ \\ Brian G Craig, ${ }^{1}$ Frank A Casey ${ }^{1}$
}

\begin{abstract}
- Additional supplemental material is published online only. To view, please visit the journal online (http://dx.doi.org/10. 1136/openhrt-2021-001599).

To cite: Callaghan S, Morrison ML, McKeown PP, et al. Exercise prescription improves exercise tolerance in young children with $\mathrm{CHD}$ : a randomised clinical trial. Open Heart 2021;8:e001599. doi:10.1136/ openhrt-2021-001599
\end{abstract}

Received 1 March 2021 Revised 9 March 2021 Accepted 31 March 2021
D Check for updates

(C) Author(s) (or their employer(s)) 2021. Re-use permitted under CC BY-NC. No commercial re-use. See rights and permissions. Published by BMJ.

${ }^{1}$ Paediatric Cardiology Department, Royal Belfast Hospital for Sick Children, Belfast, UK

${ }^{2}$ School of Medicine, Dentistry and Biomedical Sciences, Queen's University Belfast, Belfast, UK

Correspondence to Dr Sinead Callaghan; scallaghan@doctors.org.uk

\section{ABSTRACT}

Objective The main objective of this study was to ascertain if a structured intervention programme can improve the biophysical health of young children with congenital heart disease (CHD). The primary end point was an increase in measureable physical activity levels following the intervention.

Methods Patients aged 5-10 years with CHD were identified and invited to participate. Participants completed a baseline biophysical assessment, including a formal exercise stress test and daily activity monitoring using an accelerometer. Following randomisation, the intervention group attended a 1 day education session and received an individual written exercise plan to be continued over the 4-month intervention period. The control group continued with their usual level of care. After 4 months, all participants were reassessed in the same manner as at baseline.

Results One hundred and sixty-three participants (mean age 8.4 years) were recruited, 100 of whom were male $(61.3 \%)$. At baseline, the majority of the children were active with good exercise tolerance. The cyanotic palliated subgroup participants, however, were found to have lower levels of daily activity and significantly limited peak exercise performance compared with the other subgroups. One hundred and fifty-two participants (93.2\%) attended for reassessment. Following the intervention, there was a significant improvement in peak exercise capacity in the intervention group. There was also a trend towards increased daily activity levels.

Conclusion Overall physical activity levels are well preserved in the majority of young children with $\mathrm{CHD}$. A structured intervention programme significantly increased peak exercise capacity and improved attitudes towards positive lifestyle changes.

\section{INTRODUCTION}

With advances in medical and surgical interventions over the past 20-30 years, over $80 \%$ of patients with congenital heart disease (CHD) are now surviving into adulthood. ${ }^{1}$ As a result, the importance of planning for long-term physical and psychological health is becoming increasingly essential. One area which is being targeted to help improve these

\section{Key questions}

What is already known about this subject?

- Children with congenital heart disease (CHD) have been shown to have a lower level of daily activity and exercise tolerance than their peers.

- Exercise capacity in adolescents and young adults post-Fontan procedure has been shown to steeply decline in late childhood and adolescence.

- Reports suggest that exercise limitation in adults with CHD correlates poorly with both long-term morbidity and mortality.

- The role and benefits of cardiac rehabilitation in adults with ischaemic heart disease are well documented.

- There are a small number of studies, with mixed methodologies, that have previously investigated the benefit of exercise training in patients with CHD

- The numbers conducted in children are few but support the theory that training can improve exercise tolerance in younger patients with CHD.

What does this study add?

- To date, this is the largest randomised controlled trial investigating the impact of exercise training in children with $\mathrm{CHD}$.

- The younger age group involved makes it unique and for this reason the baseline results are of interest and provide important information on the biophysical health of young children with CHD.

- Importantly, this study has demonstrated that a structured exercise programme can improve peak exercise capacity in these patients.

- An important factor that makes this study distinctive is the utilisation of motivational interviewing to deliver the information during the intervention session.

- In a recent statement from the American Heart Association, the use of motivational interviewing to initiate effective behaviour change and improve the likelihood of sustaining changes is discussed in detail. $^{27}$

- The use of individual exercise prescriptions and the one-to-one counselling of the parents with a research doctor was also crucial to the success of the study. 


\section{How might this impact on clinical practice?}

- The real advantage with this study design is that many aspects can be incorporated into existing care models.

- Since this study, exercise prescription has been introduced into routine outpatient clinics.

- Providing written information to families regarding exercise advice has received very positive feedback and we would propose introducing exercise advice to nurse-led clinics as part of their regular reviews.

- In addition, motivational interviewing techniques are skills that can be used in these sessions to maximise motivation and maintenance of good habits in the future.

- The aim is that, by improving exercise tolerance and daily activities in young children with CHD and importantly maintaining it throughout adolescence, we can improve long-term physical and psychosocial outcomes in these patients.

outcomes is the patient's ability to participate in regular physical activity. Worryingly, children with CHD have been found to have lower levels of daily physical activity ${ }^{23}$ and a higher prevalence of obesity than their peers. ${ }^{4}$ In addition, exercise limitation in adults with CHD has been shown to correlate with poorer long-term outcomes. ${ }^{5}$

The role of exercise in cardiac rehabilitation for patients with ischaemic heart disease is well recognised. ${ }^{6}$ More recently, the benefit of these programmes has been demonstrated in patients with CHD. ${ }^{7}$

This study aimed to ascertain if a structured intervention programme can improve both the biophysical and psychosocial health of young children with CHD. The primary end point set as a measureable increase in physical activity following the intervention, as measured using an exercise stress test (EST) and an accelerometer. This paper reports the physical activity results.

\section{METHODS}

This study was a prospective randomised controlled trial. The research was conducted at the Department of Paediatric Cardiology, Royal Hospital for Sick Children, Northern Ireland.

\section{Patient and public involvement}

Patients were involved early in the planning stages of this study. This research team had previously conducted a study with a similar methodology. ${ }^{7}$ Participants from the previous study were sent a questionnaire asking for feedback on their experience. The questionnaire addressed information leaflets, the recruitment process, informed consent as well as the components of the intervention programme. Areas suggested for improvement included facilitating travel arrangements and attendances for appointments. We endeavoured to address this by providing travel expenses and coordinating research appointments with other hospital visits where possible. This current study was also advertised on the website and social media of a local charity, Children's Heartbeat Trust (CHT). In addition, the project was presented on several occasions at the annual CHT Parents' Conference. This included the presentation of the study results which were also published on their website.

\section{Recruitment}

Children aged 5-10 years with a diagnosis of CHD were eligible for inclusion. Patients with a cardiac diagnosis where it would be considered unsafe for them to participate in even moderate physical activity were excluded. Patients with a syndromic diagnosis, a major learning difficulty or other serious comorbidity that would either prevent or significantly hinder their participation were also excluded.

Eligible patients were identified using the Heartsuite database and contacted with an information pack either delivered by post or offered at an outpatient clinic. Informed consent was obtained at the first research appointment.

The diagnoses of the participants included a spectrum of CHD. To allow comparisons based on disease severity the participants were divided into four diagnostic categories (table 1 ).

\section{Baseline assessment}

At baseline each participant underwent a full clinical examination. All participants completed a formal EST using a GE ebike comfort cycle ergometer linked to a GE Cardiac Assessment System for Exercise (CASE V.6.61) (GE, Wauwatosa, Wisconsin, USA) (figure 1). The ebike was fitted with an adjustable paediatric saddle. The ebike was carefully adjusted for each participant. A graded ramped protocol was used which was a modified ebike protocol. Modifications were made after piloting the protocol with a number of 'heart healthy' children. The study protocol commenced with an initial workload of $25 \mathrm{~W}$. Each stage of the protocol lasted 2 min with an increasing load every stage of $15 \mathrm{~W}$ increments. Children were instructed to cycle at a rate of $60 \mathrm{rpm}$, which was represented by both numeric and light indicators on the ebike display. The 12-lead ECGs were recorded at baseline and at $2 \mathrm{~min}$ intervals during exercise and recovery. Participants were verbally encouraged to exercise until exhaustion unless there was a clinical indication to stop before this point. Following this, participants recovered for $6 \mathrm{~min}$ before ending the test.

Daily free-living activity was assessed using an Actigraph wGT3X-BT (Actigraph, Pensacola Florida) accelerometer. The Actigraph wGT3X-BT is a triple axis accelerometer that is a validated and extensively used method of measuring free-living activity in children. The device was waist-worn with an elastic belt fastening. Each device was set to collect data over a 7-day period at a sampling rate of $30 \mathrm{~Hz}$ and downloaded with epoch lengths of $60 \mathrm{~s}$ using three axis data, including step counts. Evenson cutoff points were used to define sedentary, light, moderate and vigorous activity levels. Evenson activity count cut-off points have been previously used and validated in the literature for use in children. ${ }^{8}$ Participants were required 

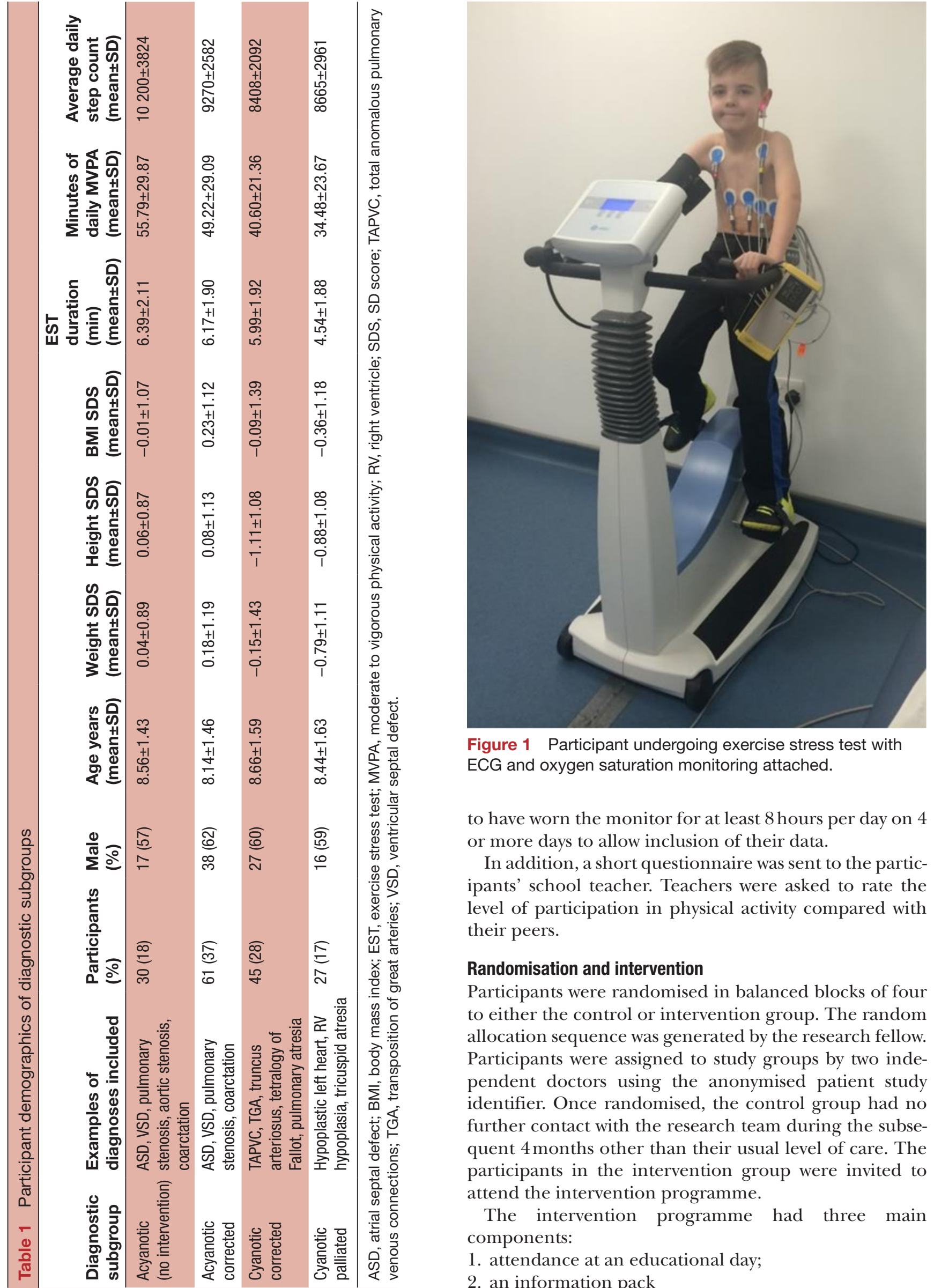

Figure 1 Participant undergoing exercise stress test with ECG and oxygen saturation monitoring attached.

to have worn the monitor for at least 8 hours per day on 4 or more days to allow inclusion of their data.

In addition, a short questionnaire was sent to the participants' school teacher. Teachers were asked to rate the level of participation in physical activity compared with their peers.

\section{Randomisation and intervention}

Participants were randomised in balanced blocks of four to either the control or intervention group. The random allocation sequence was generated by the research fellow. Participants were assigned to study groups by two independent doctors using the anonymised patient study identifier. Once randomised, the control group had no further contact with the research team during the subsequent 4 months other than their usual level of care. The participants in the intervention group were invited to attend the intervention programme.

The intervention programme had three main components:

1. attendance at an educational day;

2. an information pack 


\begin{tabular}{|c|c|}
\hline \multicolumn{2}{|l|}{ 1. Welcome and Introduction: $10.00-10.15$} \\
\hline $\begin{array}{l}\text { 2. Motivational Group Session: } \mathbf{1 0 . 1 5} \text { - } \\
\mathbf{1 1 . 1 5} \\
\text { For Parents only, delivered by Clinical Psychologist. } \\
\text { Using motivational interviewing techniques the } \\
\text { concepts of motivation and positive lifestyle change } \\
\text { were introduced. Parents were encouraged to discuss } \\
\text { perceived barriers and solutions to increasing their } \\
\text { child's activity levels. }\end{array}$ & $\begin{array}{l}\text { 2. Children's Group Session: 10.15 - } 11.15 \\
\text { Taken by Play therapist } \\
\text { Group brainstorming with crafts and art based on fun } \\
\text { sports/activities the children enjoyed. }\end{array}$ \\
\hline $\begin{array}{l}\text { 3. Parents Dietary Advice: } \mathbf{1 1 . 1 5 - 1 2 . 0 0} \\
\text { Delivered by a paediatric dietician } \\
\text { Short healthy eating quiz followed by presentation on } \\
\text { dietary advice. }\end{array}$ & $\begin{array}{l}\text { 3. Children's food session: } \mathbf{1 1 . 1 5 - 1 2 . 0 0} \\
\text { Using art, games and food demonstrations the children } \\
\text { were also educated on healthy eating and exercise. }\end{array}$ \\
\hline \multicolumn{2}{|l|}{ Lunch Break: $12.00-12.30$} \\
\hline $\begin{array}{l}\text { 4. Group } 1 \\
\text { Coaching Session: 12.30-13.15 } \\
\text { Children + Adults together } \\
\text { Led by Sports Scientist - families participated in } \\
\text { games and given examples of activities that could be } \\
\text { incorporated at home }\end{array}$ & $\begin{array}{l}\text { 4. Group } 2 \\
\text { Meeting with Doctor: 12.30-13.15 } \\
\text { Parents met with individually and given their child's } \\
\text { individualised exercise plan which was agreed upon } \\
\text { following one to one discussion between the } \\
\text { parent/guardian and a cardiologist }\end{array}$ \\
\hline $\begin{array}{l}\text { 5. Group } 2 \\
\text { Coaching Session: 13.15-14.00 }\end{array}$ & $\begin{array}{l}\text { 5. Group } 2 \\
\text { Meeting with Doctor: 13.15-14.00 }\end{array}$ \\
\hline \multicolumn{2}{|c|}{$\begin{array}{l}\text { 6. Finish and Close: } \mathbf{1 4 . 0 0 - 1 4 . 3 0} \\
\text { Sum up, further readiness ruler completed. } \\
\text { Information packs distributed (including copies of the presentations, information leaflets on diet, portion sizes, } \\
\text { suggested meal plans and family activities and an Activity Chart). }\end{array}$} \\
\hline
\end{tabular}

Figure 2 Schedule for Fit Kids for Healthy Hearts education day.

3. an individualised exercise advice plan.

During the 'Fit Kids for Healthy Hearts' education day, participants attended four main sessions (figure 2). The core component centred around a motivational group session delivered by a clinical psychologist. Finally, families met individually with a study doctor to discuss options for increasing activity levels safely over the next 4 months and each received an individualised exercise plan.

Following attendance, a letter of encouragement was sent which included a list of key elements of the motivational group session. Participants were encouraged to keep in touch by telephone, email or text with the research fellow. A summarised exercise plan was also posted to their primary school teacher to guide participation in activities during school hours. After the 4-month intervention period, all participants were reassessed in the same manner as at baseline.

\section{Statistical analysis}

The power calculation was based on the estimate that following the intervention twice as many participants would achieve the recommended daily physical activity recommendations compared with before. The power calculation was performed using StatCalc programme (EpiInfo V.7) with CIs set at $95 \%$ and $80 \%$ power. The study sample was estimated to be between 166 and 182, therefore a study population of 180 was agreed.

Study results were analysed using IBM SPSS Statistics V.22. As the data satisfied assumptions of normality, parametric methods were used. Comparisons between the diagnostic subgroups were analysed using one-way analysis of variance (ANOVA). Post hoc tests such as StudentNewman-Keuls and Fisher's least significant difference were conducted for results with a significant $\mathrm{F}$ statistic. 
Table 2 Summary of ANOVA and post hoc results for growth parameter comparisons across subgroups

\begin{tabular}{lcccc}
\hline & Mean & SD & ANOVA F & $\begin{array}{l}\text { ANOVA } \\
\text { p value }\end{array}$ \\
\hline $\begin{array}{l}\text { Weight SDS } \\
\begin{array}{l}\text { Acyanotic no } \\
\text { intervention }\end{array}\end{array}$ & 0.04 & 0.89 & 4.22 & 0.007 \\
$\begin{array}{l}\text { Acyanotic } \\
\text { corrected }\end{array}$ & 0.18 & 1.19 & & \\
$\begin{array}{l}\text { Cyanotic } \\
\text { corrected }\end{array}$ & -0.15 & 1.43 & & \\
$\begin{array}{l}\text { Cyanotic } \\
\text { palliated }\end{array}$ & -0.79 & 1.11 & & \\
\hline
\end{tabular}

\section{Height SDS}

\begin{tabular}{|c|c|c|c|c|}
\hline $\begin{array}{l}\text { Acyanotic no } \\
\text { intervention }\end{array}$ & 0.06 & 0.87 & & \\
\hline $\begin{array}{l}\text { Acyanotic } \\
\text { corrected }\end{array}$ & 0.08 & 1.13 & & \\
\hline $\begin{array}{l}\text { Cyanotic } \\
\text { corrected }\end{array}$ & -0.11 & 1.08 & & \\
\hline $\begin{array}{l}\text { Cyanotic } \\
\text { palliated }\end{array}$ & -0.88 & 1.08 & 5.61 & 0.001 \\
\hline
\end{tabular}

\begin{tabular}{lcl}
\hline Subgroup comparison & $\mathbf{P}$ value & $\mathbf{9 5 \%} \mathbf{C l}$ \\
\hline Weight SDS & & \\
\hline $\begin{array}{l}\text { Cyanotic palliated versus } \\
\text { Acyanotic no intervention }\end{array}$ & 0.01 & -1.46 to -0.20 \\
\hline Acyanotic corrected & $<0.001$ & -1.52 to -0.42 \\
\hline Cyanotic corrected & 0.03 & -1.22 to -0.07 \\
\hline Height SDS & & \\
Cyanotic palliated versus & & \\
\hline Acyanotic no intervention & 0.001 & -1.50 to -0.38 \\
\hline Acyanotic corrected & $<0.001$ & -1.45 to -0.48 \\
\hline Cyanotic corrected & 0.003 & -1.28 to -0.26 \\
\hline
\end{tabular}

ANOVA, analysis of variance; SDS, SD score.

The reassessment data postintervention was compared with the baseline findings using ANOVA with repeated measures.

\section{RESULTS}

The recruitment period ran from December 2014 to December 2015, with reassessments between June 2015 and June 2016. One hundred and sixty-three patients were recruited, 100 were male $(61.3 \%)$ and the mean age was $8.4 \pm 1.5$ years, with a range of $5.3-11.5$ years.

Age-adjusted comparisons of the growth parameters were made using SD scores (SDS) calculated from the UK90 growth reference data. ${ }^{9}$ The majority of participants were found to fall within 2 SDs of the mean. The cyanotic palliated group had significantly lower weight and height compared with the other three subgroups (table 2).

\section{Baseline activity}

One hundred and thirty-three teachers (81.6\%) responded to the activity questionnaire; of those, almost $82 \%$ stated that the child participated in physical education at the same level as their classmates or better. Only $1.5 \%$ did not take part in physical education at all. The cyanotic palliated group participants were the most significantly impaired, with over $42 \%$ reported to have a below average participation.

All 163 patients completed the EST at baseline. The cyanotic palliated group demonstrated a significantly shorter exercise duration $(\mathrm{p}=0.001)$ and lower power output $(p=0.002)$ at peak exercise compared with the other three subgroups. The other three groups were found to have comparable EST results.

All 163 participants received an Actigraph accelerometer. The mean duration of wear was $6.53 \pm 0.95$ days. Seven of the participants did not wear the monitor for the minimum required time and were excluded from the analysis. The study group overall was found to spend an average of $45 \mathrm{~min}$ in MVPA per day, with an average step count of 9101 daily. The cyanotic palliated group had a significantly lower average time spent in MVPA per day when compared with the two acyanotic subgroups (acyanotic no intervention, $\mathrm{p}<0.001$, acyanotic repaired $\mathrm{p}<0.001)$. The daily step count also appeared to decrease with increasing CHD severity but this was of borderline statistical significance $(\mathrm{p}=0.051)$ (table 1$)$.

\section{Intervention programme}

Eighty-two participants were randomised to the intervention group and 81 to the control group. There were no significant differences found between the groups in any of the demographic or biophysical variables investigated.

Seventy-three participants attended the education day. Nine participants failed to attend any of the dates offered; however, they were kept in the data analysis on an intention-to-treat basis. Six education days were held, with an average attendance of 12 participants.

Parents were also asked to complete 'readiness rating rulers', both before and after the education day. The readiness ruler is a validated tool used extensively in both clinical and research settings as a simple method for determining patients' readiness to change by asking where they are on a scale of $1-10 .{ }^{10-13}$ The lower numbers indicate less readiness, and the higher numbers indicate greater readiness for change. Parents were asked to score the importance, their confidence and their readiness to increase their child's activity levels. The results demonstrated a statistically significant increase in all three parameters following the intervention day (table 3).

\section{Reassessment}

One hundred and fifty-two participants (93.2\%) attended for follow-up assessment. There was no significant difference in the number of patients lost between the study groups (figure 3 ) or the diagnostic subgroups. 
Table 3 Statistical analysis of presession and postsession readiness rating scores

\begin{tabular}{|c|c|c|c|c|c|c|c|}
\hline & \multicolumn{2}{|c|}{ Presession } & \multicolumn{2}{|c|}{ Postsession } & \multirow[b]{2}{*}{ Mean difference } & \multirow[b]{2}{*}{$P$ value } & \multirow[b]{2}{*}{$95 \% \mathrm{Cl}$} \\
\hline & Mean & SD & Mean & SD & & & \\
\hline Importance & 7.28 & 2.33 & 8.55 & 2.46 & -1.268 & 0.002 & -2.033 to to 0.502 \\
\hline Confidence & 6.69 & 2.31 & 8.87 & 1.40 & -1.915 & $<0.001$ & -2.550 to to 1.281 \\
\hline Readiness & 7.54 & 2.33 & 9.00 & 1.40 & -1.465 & $<0.001$ & -2.093 to -0.836 \\
\hline
\end{tabular}

There was no significant difference found in any of the growth parameters following the intervention period (table 4).

All who attended for reassessment completed the follow-up EST. Participants in the intervention group were found to have a significant increase in exercise duration $(\mathrm{p}<0.001)$ at follow-up compared with the control group. The estimated effect size calculated was a $43 \mathrm{~s}$ increase in the intervention group, which was an increase on baseline time of over $11 \%$. Similarly, there was also a significant increase in the maximum power output during the EST $(\mathrm{p}<0.001)$ in the intervention group (table 4, figure 4).

One hundred and fifty patients received an accelerometer at reassessment and of these 13 were excluded from the analysis due to insufficient wear time. The Actigraph reassessment had a mean wear time of 6.4 days (SD 1.03). The data demonstrated a trend towards a larger increase in daily activity among the intervention group compared with the controls. This was evident in both the average number of steps per day and the time spent in MVPA per

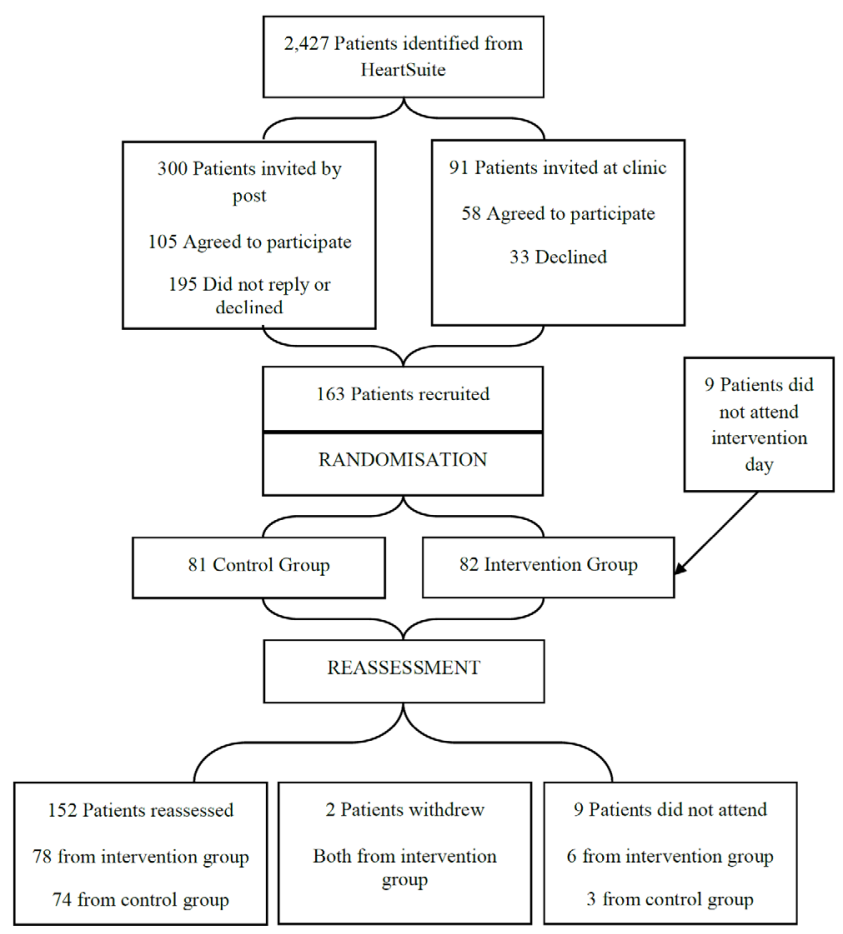

Figure 3 Consolidated Standards of Reporting Trials flow diagram demonstrating participant progression through the study. day. This difference, however, failed to reach statistical significance (table 4 , figure 4 ).

\section{DISCUSSION}

With improved survival and a rapidly growing adult population, it is crucial that the goals of long-term CHD management expand beyond the cardiovascular impact of the disease. This study adds to the growing body of evidence supporting the use of exercise training in CHD.

The baseline assessments suggest that daily activity levels in young children with CHD are well preserved. The acyanotic, no intervention group had an average daily MVPA of $56 \mathrm{~min}$, which is encouraging given the current recommendations for this age group is a minimum of $60 \mathrm{~min} /$ day. Studies have found that as little as $20 \%$ of children in Northern Ireland are currently meeting this target, ${ }^{14}$ suggesting this group appears to be at least as active as their peers. These results are supported by recent studies that have demonstrated children and teenagers with CHD have daily MVPA levels that are comparable to that of the general population. ${ }^{15} 16$ It is well recognised that many of the barriers to physical activity in CHD are similar to the well-established risk factors for inactivity in childhood in the general population. Research has shown that variables such as higher family income, less weekday time spent outdoors, the child's perception of barriers to activity and a history of a learning difficulty, are all associated with lower levels of daily activity. ${ }^{4}$ In particular, children having their activity levels unnecessarily limited due to parental or carer anxiety is an important contributor that this study aimed to address. Interestingly, it has also been postulated that the more recent reports of comparable activity levels in CHD may be as a result of current recommendations being more widely implemented within specialist cardiac services. ${ }^{16}$ Promoting physical activity and regular exercise has been a high priority for this Cardiology Department for a number of years and these results may well reflect some of that work also.

Importantly, however, the cyanotic palliated subgroup were found to be significantly more limited in daily MVPA (34.48 $\pm 23.67 \mathrm{~min})$ and at peak exercise (EST duration $4.54 \pm 1.88 \mathrm{~min}$ ), their average daily step count was just below the overall group average (9101 steps) at 8665 steps. This suggests that while children with complex CHD are more limited at vigorous activity, they remain active at lower intensity exercises. The significant differences found in the cyanotic palliated group are consistent with 
Table 4 Characteristics of study groups at baseline and comparison of activity results between study groups at reassessment

\section{A: Characteristics of study groups at baseline}

\begin{tabular}{llll}
\hline & Intervention group $(\mathbf{n}=\mathbf{8 2})$ & Control group (n=81) & P value \\
\hline Mean age & 8.50 & 8.32 & 0.45 \\
\hline Number of males & $50(60.9 \%)$ & $50(61.7 \%)$ & 0.92 \\
\hline Mean BMI SDS & -0.06 & 0.06 & 0.53 \\
Mean height SDS & -0.17 & -0.10 & 0.69 \\
\hline Mean weight SDS & -0.18 & -0.02 & 0.41 \\
\hline Diagnostic subgroup & & & 0.69 \\
$\quad$ Acyanotic no intervention & $17(20.7 \%)$ & $13(16.0 \%)$ & \\
Acyanotic corrected & $30(36.6 \%)$ & $31(38.3 \%)$ & \\
Cyanotic corrected & $20(24.4 \%)$ & $25(30.9 \%)$ & \\
Cyanotic palliated & $15(18.3 \%)$ & $12(14.8 \%)$ & 0.66 \\
Participation in PE & & & \\
Above average & $6(9.1 \%)$ & $6(9.1 \%)$ & \\
Same as & $47(70.1 \%)$ & $47(71.2 \%)$ & \\
Same as with breaks & $2(3 \%)$ & $2(3 \%)$ & \\
Below average & $12(17.9 \%)$ & $2(13.6 \%)$ & \\
Not at all & 0 & $2(3 \%)$ & \\
\hline
\end{tabular}

B: Comparison of activity results between study groups at reassessment

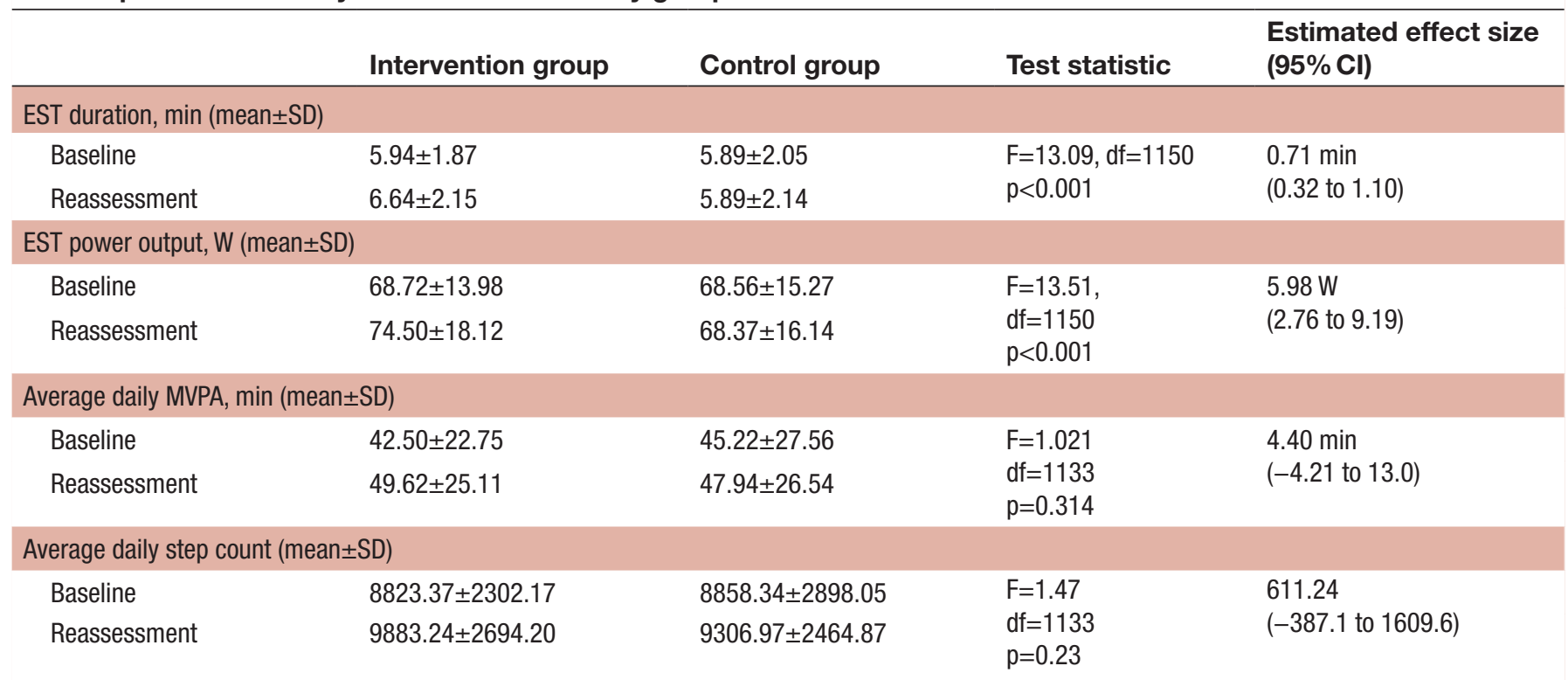

BMI, body mass index; EST, exercise stress test; MVPA, moderate to vigorous activity; PE, peak exercise; SDS, SD score.

previous research. ${ }^{2416}$ This evidence highlights an at-risk group whose low levels of physical activity in early childhood may impede their physical, cognitive and psychosocial development. The importance of this has been further highlighted with the recognition that patients whose Fontan circulations fail are those with worse ventricular function and worse exercise capacity. ${ }^{17}$ Given the limited options available to this group of patients, interventions to improve cardiopulmonary fitness are worth consideration. Even children following repaired CHD with no residual defects, however, have been found to have lower levels of aerobic fitness that can only be attributed to deconditioning, ${ }^{18}$ and would also benefit from early identification and intervention.

This study demonstrates that a structured intervention programme can significantly improve peak exercise capacity in young children with CHD. The $11 \%$ increase in EST duration seen in the intervention group was associated with a significant increase in maximal power output. These findings are in keeping with other research undertaken in older age groups. ${ }^{36719-21}$ 

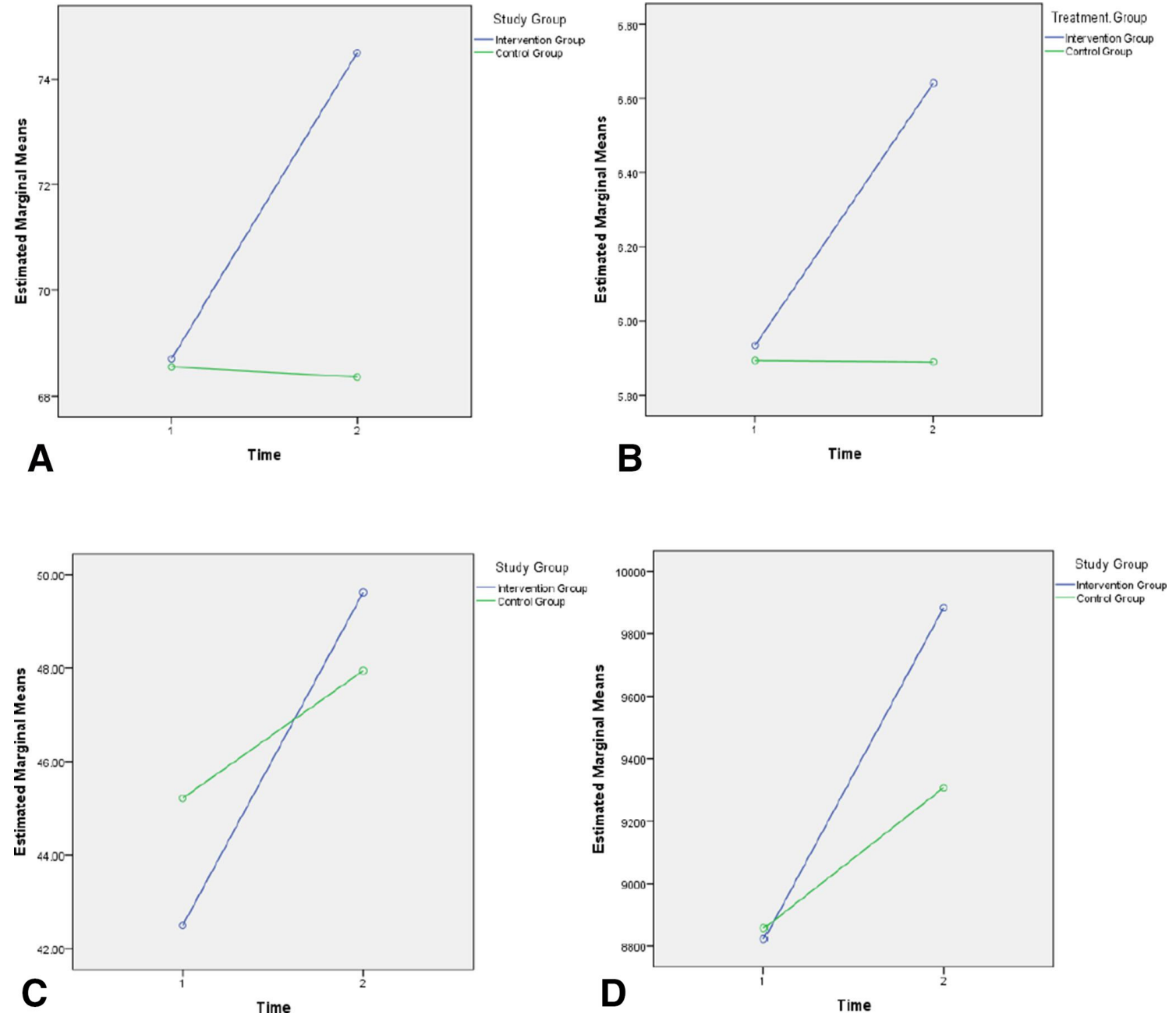

Figure 4 Marginal means plots demonstrating (A) maximum power output (W), (B) exercise stress test duration (min), (C) average daily moderate to vigorous activity (min), (D) average daily step count at baseline and reassessment for both study groups.

Reassessment results failed to demonstrate a significant increase in daily MVPA following the intervention. This may have been due to the younger study population as the majority of participants were found to have very acceptable levels of daily activity already at baseline. While adolescents with CHD have been shown to significantly increase daily activity levels following exercise training, ${ }^{721}$ it appears that, at younger ages, baseline daily activity levels offer less room for improvement as children already appear to be as active as their condition allows.

Two of the key components in this intervention programme were first, the use of an individualised exercise prescription, and second the opportunity to discuss this one to one with a paediatric cardiologist. Individualised exercise prescription in CHD has been receiving increasingly more interest in recent years. ${ }^{22}{ }^{23}$ Previous research has demonstrated that individuals are often unaware of what level and types of exercise are safe for them and many indicated that they would appreciate more information from their healthcare providers. ${ }^{24} \mathrm{~A}$ recent survey revealed that only $21 \%$ of clinicians always discuss exercise with patients during clinics, with $36 \%$ admitting to never providing written advice. ${ }^{25}$ The opportunity parents had to discuss concerns and their child's individual exercise plan proved to be a crucial part of the intervention. Indeed, this session scored the highest on parental feedback. Given that it has been previously demonstrated a mother's overprotective nature is a key factor that can negatively influence the child's activity levels, ${ }^{26}$ positive counselling by the cardiologist has the potential to make a significant impact.

An important factor that makes this study distinctive is the utilisation of motivational interviewing during the intervention programme. This session was found to have a positive effect on parents' attitudes to exercise, as evidenced by the readiness rating scores. The benefits of using motivational interviewing in physical activity counselling and promotion in CHD are now becoming increasingly recognised. ${ }^{27}$

The convenience of this home-based exercise programme is likely to have been particularly appealing. The exercise plans were also devised to allow participants the choice of a variety of activities. The flexibility offered by this programme may increase the chances of these positive lifestyles changes being maintained in the future. 
No serious adverse events occurred during the implementation of this research study. This adds further support to previous conclusions that exercise training is safe for the majority of individuals with CHD. ${ }^{28}$

\section{Study limitations}

Due to the 2-year time frame for the completion of this study, recruitment was limited to a 12-month period. Despite best efforts recruitment fell short of the original 180 target by 17 participants. The attrition rate, however, was less than expected at $6.7 \%$.

There is no doubt that this study required substantial effort on behalf of the families who took part and was stated to be a factor for some who opted not to take part.

\section{Future considerations}

This study supports the role of cardiac rehabilitation even in this much younger age group. However, further work is needed. The primary outcome measures for this study focused on the impact of exercise training on peak exercise capacity as assessed by a formal EST and daily activity levels, measured by an accelerometer. Comparisons across previous studies are made difficult due to different end points being investigated. ${ }^{3721}$ Significant differences also exist in the format of the exercise training implemented. While both home and supervised exercise programmes have been demonstrated to be successful, ${ }^{28}$ further evidence is required to ascertain which method is more feasible for long-term maintenance. Ongoing encouragement and reinforcement of these lifestyle changes is vital in order to sustain health benefits and long-term outcomes. Prior to future research, issues regarding primary outcomes measures, type, intensity and duration of training and training location all need to be addressed. Consideration should also be given to investigating the effects of exercise training on cardiac function in individuals with CHD through the use of echocardiogram or cardiac MRI. A recent Cochrane review on physical activity interventions in CHD concluded that while there have been small and modest improvements demonstrated with exercise training, there is still little evidence on the long-term impact this has on prognosis. ${ }^{29}$ In addition to the need for a larger multicentre RCT to investigate the impact of exercise training on cardiopulmonary fitness in CHD, further research is required to assess the impact in the long term on morbidity, mortality, psychosocial wellbeing and quality of life.

\section{CONCLUSION}

This study has made an important contribution to the work already undertaken in this field. It has provided evidence that, in the majority of young children living with CHD, physical activity levels are well preserved. Importantly, a structured exercise programme can improve attitudes towards making positive lifestyle choices and, in turn, increases peak exercise abilities in even young children with CHD.
Twitter Sinead Callaghan @dr_scallaghan

Acknowledgements The authors gratefully acknowledge statistical advice provided by Dr Chris Patterson. The authors acknowledge the important contribution of the support staff that facilitated the intervention days. Finally, the authors want to thank all the families who participated in the study.

Contributors FAC, MLM: concept, study design and revision of manuscript. SC: study design, recruitment, data collection, analysis and interpretation, manuscript preparation and revision. PPMcK: study design and revision of manuscript. CT: psychological support. AJS: recruitment and revision of manuscript. BMcC, BG, BGC: recruitment.

Funding This study was funded by a Research Fellowship from the RBHSC Medical Staff Committee and supported by the Children's Heartbeat Trust.

Competing interests None declared.

Patient consent for publication Parental/guardian consent obtained.

Ethics approval Ethical approval was granted by Wales Research Ethics Committee 5, Research Ethics Service (Ref:14/WA/0131).

Provenance and peer review Not commissioned; externally peer reviewed.

Data availability statement De-identified participant data in form of Excel and SPSS data sheets are available from the research team on reasonable request. This is available from the first author Dr Sinead Callaghan (scallaghan@doctors. org.uk).

Open access This is an open access article distributed in accordance with the Creative Commons Attribution Non Commercial (CC BY-NC 4.0) license, which permits others to distribute, remix, adapt, build upon this work non-commercially, and license their derivative works on different terms, provided the original work is properly cited, appropriate credit is given, any changes made indicated, and the use is non-commercial. See: http://creativecommons.org/licenses/by-nc/4.0/.

ORCID iD

Sinead Callaghan http://orcid.org/0000-0002-6849-1294

\section{REFERENCES}

1 Ehlert N, Hess J, Hager A. Shifts in exercise capacity are not reported adequately in patients with congenital heart disease. Congenit Heart Dis 2012;7:448-54.

2 McCrindle BW, Williams RV, Mital S, et al. Physical activity levels in children and adolescents are reduced after the Fontan procedure, independent of exercise capacity, and are associated with lower perceived general health. Arch Dis Child 2007;92:509-14.

3 Rhodes J, Curran TJ, Camil L, et al. Impact of cardiac rehabilitation on the exercise function of children with serious congenital heart disease. Pediatrics 2005;116:1339-4.

4 Longmuir PE, Russell JL, Corey M, et al. Factors associated with the physical activity level of children who have the Fontan procedure. Am Heart J 2011;161:411-7.

5 Diller G-P, Dimopoulos K, Okonko D, et al. Exercise intolerance in adult congenital heart disease: comparative severity, correlates, and prognostic implication. Circulation 2005;112:828-35.

6 Sandercock G, Hurtado V, Cardoso F. Changes in cardiorespiratory fitness in cardiac rehabilitation patients: a meta-analysis. Int $J$ Cardiol 2013;167:894-902.

7 Morrison ML, Sands AJ, McCusker CG, et al. Exercise training improves activity in adolescents with congenital heart disease. Heart 2013;99:1122-8.

8 Trost SG, Loprinzi PD, Moore R, et al. Comparison of accelerometer cut points for predicting activity intensity in youth. Med Sci Sports Exerc 2011;43:1360-8.

9 Cole TJ, Freeman JV, Preece MA. British 1990 growth reference centiles for weight, height, body mass index and head circumference fitted by maximum penalized likelihood. Stat Med 1998;17:407-29.

10 Miller WR. Motivation for treatment: a review with special emphasis on alcoholism. Psychol Bull 1985;98:84-107.

11 Hesse $M$. The readiness ruler as a measure of readiness to change poly-drug use in drug abusers. Harm Reduct $J$ 2006;3:3.

12 Maisto SA, Krenek M, Chung T, et al. A comparison of the concurrent and predictive validity of three measures of readiness to change alcohol use in a clinical sample of adolescents. Psychol Assess 2011;23:983-94.

13 St-Hilaire A, Axelrod K, Geller J, et al. A readiness ruler for assessing motivation to change in people with eating disorders. Eur Eat Disord Rev 2017;25:417-22. 
14 Townsend N, Bhatnagar P, Wickramasinghe K. Children and young people statistics. In: Weissberg P, ed. London: British Heart Foundation, 2013.

15 Voss C, Duncombe SL, Dean PH, et al. Physical activity and sedentary behavior in children with congenital heart disease. J Am Heart Assoc 2017;6:e004665

16 Brudy L, Hock J, Häcker A-L, et al. Children with congenital heart disease are active but need to keep moving: a cross-sectional study using Wrist-Worn physical activity Trackers. J Pediatr 2020;217:13-19.

17 d'Udekem Y, Fitness C. Not the severity of the condition, dictates late outcomes after Fontan procedures. J Am Coll Cardiol 2017;69:2745-7.

18 Rosenblum O, Katz U, Reuveny R, et al. Exercise performance in children and young adults after complete and incomplete repair of congenital heart disease. Pediatr Cardiol 2015;36:1573-81.

19 Rhodes J, Curran TJ, Camil L, et al. Sustained effects of cardiac rehabilitation in children with serious congenital heart disease. Pediatrics 2006;118:e586-93.

20 Longmuir PE, Tremblay MS, Goode RC. Postoperative exercise training develops normal levels of physical activity in a group of children following cardiac surgery. Pediatr Cardiol 1990;11:126-30.

21 Fredriksen PM, Kahrs N, Blaasvaer S, et al. Effect of physical training in children and adolescents with congenital heart disease. Cardiol Young 2000;10:107-14.

22 Budts W, Börjesson M, Chessa M, et al. Physical activity in adolescents and adults with congenital heart defects: individualized exercise prescription. Eur Heart J 2013;34:3669-74.

23 Takken T, Giardini A, Reybrouck T, et al. Recommendations for physical activity, recreation sport, and exercise training in paediatric patients with congenital heart disease: a report from the Exercise, Basic \& Translational Research Section of the European Association of Cardiovascular Prevention and Rehabilitation, the European Congenital Heart and Lung Exercise Group, and the Association for European Paediatric Cardiology. Eur J Prev Cardiol 2012;19:1034-65.

24 Kendall L, Parsons JM, Sloper P, et al. A simple screening method for determining knowledge of the appropriate levels of activity and risk behaviour in young people with congenital cardiac conditions. Cardiol Young 2007;17:151.

25 Williams CA, Gowing L, Horn R, et al. A survey of exercise advice and recommendations in United Kingdom paediatric cardiac clinics. Cardiol Young 2017;27:951-6.

26 Bar-Mor G, Bar-Tal Y, Krulik T, et al. Self-efficacy and physical activity in adolescents with trivial, mild, or moderate congenital cardiac malformations. Cardiol Young 2000;10:561-6.

27 Longmuir PE, Brothers JA, de Ferranti SD, et al. Promotion of physical activity for children and adults with congenital heart disease: a scientific statement from the American Heart Association. Circulation 2013;127:2147-59.

28 Duppen N, Takken T, Hopman MTE, et al. Systematic review of the effects of physical exercise training programmes in children and young adults with congenital heart disease. Int $J$ Cardiol 2013;168:1779-87.

29 Williams CA, Wadey C, Pieles G, et al. Physical activity interventions for people with congenital heart disease. Cochrane Database Syst Rev 2020;10:CD013400. 